\title{
Pratiques
}

Linguistique, littérature, didactique

\section{Genres littéraires et genres textuels dans la discipline français}

Nathalie Denizot

\section{(2) OpenEdition}

1 Journals

Édition électronique

URL : http://journals.openedition.org/pratiques/1562

DOI : 10.4000/pratiques.1562

ISSN : 2425-2042

Éditeur

Centre de recherche sur les médiations (CREM)

Édition imprimée

Date de publication : 15 juin 2010

Pagination : 211-230

\section{Référence électronique}

Nathalie Denizot, "Genres littéraires et genres textuels dans la discipline français », Pratiques [En ligne], 145-146 | 2010, mis en ligne le 15 juin 2010, consulté le 14 novembre 2019. URL : http:// journals.openedition.org/pratiques/1562 ; DOI : 10.4000/pratiques.1562

(c) Tous droits réservés 


\title{
Genres littéraires et genres textuels dans la discipline français
}

\author{
Nathalie Denizot
}

Théodile-CIREL (É.A. 4354), Lille 3

Cet article se propose de présenter les principaux résultats d'une recherche doctorale (Denizot, 2008) ${ }^{(1)}$ sur la scolarisation des genres littéraires et textuels. J'ai en effet interrogé les relations que la discipline français entretient avec ces objets d'enseignement et d'apprentissage que sont les genres littéraires, pour comprendre quels sont leurs modes de scolarisation, et quels sont leurs usages et leurs fonctions dans la discipline. Et comme le français a connu des variations importantes, j'ai donné à mon travail une dimension diachronique, en interrogeant 1 'histoire de la discipline, de 1802 (date de création des lycées impériaux) à 2008, avec une périodisation - classique - en trois grands moments ${ }^{(2)}$ : une première configuration prédisciplinaire $^{(3)}$, entre 1802 et 1880 , est marquée par l'enseignement de la rhétorique, avec des pratiques d'écriture spécifiques; une seconde configuration, chevauchant en partie la première, entre 1860 et 1960, est marquée par l'affaiblissement de la rhétorique au profit de la poétique et de l'histoire littéraire, et s'articule autour de nouvelles pratiques de lecture et de commentaire des textes; la troisième, de 1960 à aujourd'hui, a vu le développement des sciences humaines introduire de nouveaux objets de travail et de nouvelles pratiques disciplinaires. Cette périodisation sert notamment de cadre à une sorte de cartographie scolaire de la notion de « genre » depuis 1802. Mais cette thèse est une recherche didactique et non pas historique : après une étude des formes scolaires du genre dans la discipline depuis 1802, j'ai mis en perspective la notion de genre avec d'autres modes de classement des textes, dans la discipline mais aussi à ses frontières (notamment dans les CDI). Je me suis ensuite attachée aux modes de construction des genres à travers trois études de cas : un genre très récent au lycée et déjà disparu, le biographique ; un genre qui me sem-

(1) Sous la direction d'Yves Reuter, à qui je réitère ici mes remerciements les plus chaleureux.

(2) Les périodes peuvent se chevaucher, tant il est difficile en ce qui concerne l'histoire des disciplines de dire à quel moment précis telle finalité, telle pratique, tel exercice, etc., apparaissent et/ou disparaissent. Les textes officiels indiquent ce qui devient justement officiel à telle ou telle date, qui ne coïncide pas nécessairement avec la réalité de toutes les classes à telle ou telle date.

(3) J'utilise ce terme de prédisciplinaire pour désigner la période précédant l'invention de la littérature comme discipline, à partir de 1880 ( $c f$. Jey, 1998). 
ble en voie de construction au collège, les textes dits « fondateurs »; enfin, un genre qui semble stable et stabilisé depuis longtemps, la tragédie classique. Pour terminer, j'ai étudié les usages disciplinaires des genres, à travers deux pratiques fondamentales de la classe de français : la lecture et l'écriture. Concernant la lecture, il me semblait intéressant d'examiner ce que l'on fait en classe d'un genre comme le roman lorsqu' on lit des extraits de roman : j'ai centré mon propos sur une œuvre romanesque, celle de Balzac, entré dans l'enseignement secondaire en même temps que le roman, dans les années 1880 . Concernant les pratiques d'écriture, j'ai choisi d'étudier ce que l'on fait des genres dans l'écriture d'invention, puisque cette catégorie d'exercices, qui est l'héritière de pratiques parfois anciennes, s'articule assez fortement avec les genres lus et étudiés dans la classe.

Les corpus sur lesquels s'appuie cette recherche sont de natures diverses : textes officiels et institutionnels publiés depuis $1802^{(4)}$, manuels scolaires représentatifs et/ou atypiques (près de 200 manuels et ouvrages méthodologiques consultés, sur une période de près de deux siècles et demi), discours sur la discipline, enquêtes par questionnaires auprès d'enseignants et/ou de documentalistes et corpus de 75 copies de baccalauréat de la session 2005 .

J'ai choisi dans cet article de me centrer tout d'abord sur cette question de la construction des genres, qui me semble essentielle pour comprendre la fabrication scolaire des savoirs. Je m'attacherai plus particulièrement à analyser les processus de scolarisation des genres, en m'appuyant sur un certain nombre d'exemples variés ${ }^{(5)}$. Dans un second temps, je m'attarderai - à l'aide de quelques exemples encore - sur un concept qui me semble intéressant pour analyser les représentations des genres construites notamment dans les manuels et les groupements de textes, l'amphitextualité, qui désigne un mode particulier de solidarités textuelles.

\section{Les genres : des constructions disciplinaires}

Ce sont des genres très divers qui sont au centre de mon travail ${ }^{(6)}$ : roman et roman réaliste, théâtre et tragédie classique, genres biographiques et autobiographiques, épopée et textes bibliques, mais aussi discours, narration, etc. Ce ne sont cependant pas ces genres en tant que tels qui m'ont intéressée, mais leur forme scolaire, leur version ou leur variante scolaire, en quelque sorte. En effet, ce n'est pas par exemple le théâtre qui est à l'école objet d'enseignement, mais un théâtre scolaire, c'està-dire scolarisé, qui répond à des finalités disciplinaires particulières, de même que les discours ou les récits lus et/ou produits à l'école sont reconstruits pour devenir objets scolaires. Et ces genres scolaires sont eux-mêmes des catégories soumises à des variations synchroniques et diachroniques, ce qui m'a conduite à montrer qu'il y a non pas un mais des théâtres scolaires, par exemple, de même qu'il y a non pas une mais des vulgates balzaciennes, reconfigurées dans des configurations ${ }^{(7)}$ et pour des usages disciplinaires spécifiques.

(4) J'ai consulté quasi systématiquement la plupart des programmes et instructions officielles de l'enseignement secondaire depuis cette période. Les références précises se trouvent en bibliographie de ma thèse, ainsi que celles des manuels consultés.

(5) Pour des analyses plus développées de ces exemples, je renvoie le lecteur à ma thèse ou, le cas échéant, à certains de mes articles qui peuvent constituer des sortes de monographies.

(6) J'ai adopté une définition souple de la notion, ne prenant pas en compte les hiérarchisations possibles : genres ou sous-genres sont pour moi des genres, et je prends acte de 1'hétérogénéité constitutive de la notion. Je rejoins sur ce point Karl Canvat (1998, p. 274) pour qui c'est la faible consistance théorique de la notion qui en fait justement l'intérêt.

(7) Je reviens en conclusion sur ce concept, que j'emprunte à Lahanier-Reuter et Reuter (2007). 
Les genres sont donc des constructions disciplinaires ou prédisciplinaires, qui servent différentes finalités disciplinaires et/ou scolaires ${ }^{(8)}$ selon les configurations disciplinaires et/ou historiques : former les classes dirigeantes d'autrefois par des genres scripturaux spécifiques, créés à partir de l'héritage rhétorique ; rassembler les élèves actuels autour d'une culture fondatrice commune, à travers la lecture d'un corpus en voie de stabilisation, les « textes fondateurs » (Denizot, 2006a); sélectionner des Balzac ou des Racine « enseignables », qui puissent, selon les configurations disciplinaires, servir de modèles d'écriture ou de lectures édifiantes, ou servir à construire des savoirs culturels (littéraires ou d'histoire littéraire notamment) jugés indispensables; etc. Constructions de genres scripturaux (la « narration » scolaire, l'écriture d'invention), de genres littéraires (le « roman réaliste », la « tragédie classique ») ou reconstruction de corpus génériques (le roman balzacien ou la tragédie racinienne scolarisés à telle ou telle époque), tous ces objets disciplinaires sont devenus des objets autonomes, dont l'objectif n'est pas nécessairement de vulgariser des savoirs savants, mais de servir des finalités proprement scolaires ${ }^{(9)}$.

Je m'inscris ainsi dans un cadre théorique inspiré des travaux de Chervel (1988/1998), qui pose en effet que les disciplines d'enseignement sont des créations originales de l'école avec une double finalité : instruire mais aussi acculturer les jeunes générations, et qu'elles fonctionnent donc comme des "médiatisations » «mise [s] au service de la jeunesse scolaire dans sa lente progression vers la culture de la société globale» (p. 33); il ajoute que cette culture scolaire tend à s'autonomiser pour devenir une culture spécifique et originale, «qui ne manque pas d'investir à son tour », dit-il (2005, p. 84), la culture de la société globale. C'est cette entrée par les finalités disciplinaires qui m'a permis de décrire et d'analyser des phénomènes comme la reconfiguration des genres rhétoriques en genres disciplinaires, que je vais développer ci-après, ainsi que celle des « textes anciens » en " textes fondateurs » (voir aussi Denizot, 2009a) ; c'est aussi dans ce cadre que j'ai analysé la scolarisation des romans de Balzac (voir aussi Denizot, 2010) ou des tragédies classiques, scolarisation qui ne peut être réduite à une transposition tant elle conduit à reconstruire les corpus au gré des configurations disciplinaires, comme je le montrerai également.

\subsection{La scolarisation : un travail disciplinaire}

J'appelle « scolarisation », à la suite de Kuentz (1972) et de Chervel (2006), les phénomènes de constructions d'objets scolaires à partir d'objets extrascolaires déjà existants. J'entends ce terme comme un terme général, non marqué axiologiquement (à la différence de Kuentz), et recouvrant aussi bien des processus transpositifs que des phénomènes de secondarisation, de reconfiguration, voire de véritable création. La scolarisation s'accompagne d'un travail disciplinaire spécifique pour

(8) Des finalités comme l'éducation morale ou la formation d'une culture ne sont pas propres à la discipline, mais plutôt à certaines époques et à ce qu'on pourrait appeler des « configurations scolaires ".

(9) Qui peuvent d'ailleurs ensuite informer la culture de la société globale : on connaît par exemple 1'histoire de la réception des auteurs tragiques, que rappelle Chervel (2005), soulignant que nous ne connaissons plus désormais de l'œuvre d'Eschyle que les 7 pièces (sur près de 80 ) autrefois réunies dans un recueil à usage scolaire, qui a protégé et sauvé une partie de l'œuvre d'Eschyle en même temps qu'il a contribué à la filtrer. De la même manière, et même si l'œuvre complète d'un romancier classique comme Balzac est actuellement facilement accessible, il n'en reste pas moins que les romans les plus répandus - et donc les plus connus - sont ceux qui appartiennent à la culture scolaire : il n'est pas forcément facile de trouver des éditions isolées et bon marché des romans (ou nouvelles) moins célèbres. 
adapter les genres à la forme scolaire et disciplinaire - les scolariser - et ce travail peut prendre plusieurs aspects, qui ne sont en rien exclusifs les uns des autres, mais qui au contraire sont constamment intriqués.

\subsubsection{Sélection et vulgates scolaires : la tragédie classique}

On peut tout d'abord relever un travail de sélection, qui opère notamment sur les corpus de textes, et qui a été souvent évoqué en ce qui concerne les « morceaux choisis »; la sélection fonctionne également comme un filtre, qui ne retient que ce qui sert les finalités disciplinaires. Je prendrai ici l'exemple de la tragédie classique, genre qui semble pourtant bien stabilisé à l'école, et depuis longtemps. L'étude des corpus scolaires de la tragédie montre en effet combien chaque époque a construit et reconstruit «sa » tragédie classique, donnant ainsi naissance à plusieurs vulgates scolaires, mouvantes selon les usages disciplinaires ${ }^{(10)}$. La première période $(1802$ 1880) a construit un double corpus : d'une part des discours et morceaux oratoires extraits de tragédies variées et destinés à servir de modèles d'écriture ${ }^{(11)}$; d'autre part les trois « tragédies saintes » (Polyeucte, Esther et Athalie), pièces édifiantes également destinées à la lecture, dont l'usage est plutôt à chercher du côté des finalités morales de la discipline et dans sa construction d'un corpus d'auteurs français capables de rivaliser avec les auteurs antiques (Chervel, 2006). La configuration disciplinaire qui se met en place au tournant des $\mathrm{XIX}^{\mathrm{e}}$ et $\mathrm{XX}^{\mathrm{e}}$ siècles privilégie quant à elle un corpus de morceaux à lire et à admirer, davantage tourné vers les grands sentiments : passions raciniennes et idéaux cornéliens. Le corpus tragique dans les manuels change donc : les extraits de tragédies sélectionnés sont plus souvent des passages dialogués, ce qui minore la place des tirades et des monologues. Et, sous l'influence de l'historicisation de la littérature, le corpus se restreint encore : la tragédie classique devient la tragédie de l'époque classique, réduite à une petite partie de la seconde moitié du XVII ${ }^{\mathrm{e}}$ siècle. Quant au corpus scolaire actuel, il met davantage en valeur l'intertextualité de la tragédie classique, en la faisant dialoguer avec les auteurs tragiques antiques ainsi qu'avec le tragique du $\mathrm{XX}^{\mathrm{e}}$ siècle. Dans ce cadre, certaines pièces sont privilégiées, notamment Phèdre, à la fois mythologique et tragique.

Pourtant, comme Georges Forestier (2003, p. 303 sqq.) l'a montré, cette lecture «tragique » de la tragédie, qui conduit les spectateurs et les lecteurs «à méditer sur le drame de l'homme affronté à ce qui le passe, sur le mystère de la transcendance, ou sur la difficulté de vivre dans un système de contradictions existentielles » (id., p. 304), est une lecture moderne qui ne va pas de soi : la notion de tragique telle qu'elle est actuellement définie est inconnue des auteurs de tragédie classique, pour qui le tragique est simplement un adjectif qui se rapporte à la tragédie. Le tragique en tant que concept est une notion socio-historiquement construite, inventée à partir des tragédies grecques par des écrivains et philosophes allemands au tournant des $\mathrm{XVIII}^{\mathrm{e}}$ et XIX ${ }^{\mathrm{e}}$ siècles, et reprise par les essayistes français du $\mathrm{XX}^{\mathrm{e}}$ siècle, qui puisent chez Racine. Le tragique racinien ne correspond donc à notre conception moderne du tragique que par ce que Forestier (id., p. 312) nomme une « illusion rétrospective »- qui d'ailleurs éclaire sans doute les phénomènes de sélection du corpus scolaire actuel.

(10) J'ai étudié par ailleurs la construction de vulgates balzaciennes selon les configurations disciplinaires (Denizot, 2009b et 2010).

(11) C'est Batteux qui a montré l'exemple, dans son recueil de 1780, plusieurs fois réédité dans la première moitié du XIX ${ }^{\mathrm{e}}$ siècle. Il y fait appel à 56 pièces de 19 auteurs tragiques différents. Le recueil de Noël et Delaplace $(1804 / 1805)$ poursuit cette tradition et, sur 51 « discours et morceaux oratoires » du volume poésie, propose 43 extraits de tragédies. 
L'école construit donc des vulgates, réduisant peu à peu les auteurs de tragédies classiques aux deux seuls Corneille et Racine, et à une dizaine de pièces, et sélectionnant par exemple tantôt un Racine rhéteur, tantôt un Racine édifiant, tantôt un Racine " peintre des passions ", tantôt un Racine « tragique », le même extrait pouvant être convoqué pour illustrer l'une ou l'autre de ces facettes du genre.

\subsubsection{Syncrétisation et redéfinition}

À ce travail de sélection s'ajoute un travail de syncrétisation, qui conduit à amalgamer des cadres et des traditions parfois diverses, pour construire un nouveau paradigme disciplinaire : le modèle scolaire du genre emprunte, selon les époques, autant à ces cadres traditionnels que sont la rhétorique et la poétique qu'à l'histoire littéraire ou aux sciences du langage. La définition scolaire du genre dans les programmes les plus récents prend ainsi en compte les définitions linguistiques et discursives des genres, notamment des genres du discours, avec une référence assez nettement bakhtinienne, ce qui permet d'intégrer les genres argumentatifs. Mais en même temps, les programmes conservent malgré tout les cadres traditionnels issus de la poétique : c'est ainsi que subsiste toujours le modèle de la «triade » (épique, lyrique et dramatique, reformulé pour intégrer les genres de la prose en roman, poésie et théâtre), qui, en se calquant sur la tradition rhétorique des trois genres (judiciaire, délibératif et démonstratif), est devenu au XIX ${ }^{\mathrm{e}}$ siècle un cadre essentiel pour catégoriser les genres dans les ouvrages scolaires ; c'est ainsi que persiste aussi l'ancrage dans l'histoire littéraire, qui caractérisait les définitions des genres dans les programmes tout au long $\mathrm{du} \mathrm{XX}^{\mathrm{e}}$ siècle. Le genre, catégorie de la rhétorique, puis de la poétique, est devenu une catégorie linguistique sans que ces composantes soient exclusives les unes des autres.

Le travail de syncrétisation peut également concerner des exercices et des pratiques disciplinaires ${ }^{(12)}$ : on voit naître par exemple au début du $\mathrm{XX}^{\mathrm{e}}$ siècle un nouveau genre disciplinaire (la dissertation « atténuée » des instructions de 1909), amalgamant pratique d'écriture hypertextuelle (rhétorique) et pratiques d'écriture métatextuelle (dissertative), qui a été récemment reconstruit dans certaines pratiques d'écriture d'invention (Daunay, 2003). Ce travail de syncrétisation n'est jamais réellement achevé, les formes scolaires du genre se renouvelant régulièrement.

Cette syncrétisation s'accompagne parfois d'un travail de redéfinition qui peut aller jusqu'à l'occultation de l'ancien sens par le nouveau : la "narration » rhétorique est devenue « récit» au terme d'un long processus de digestion ( $c f$. infra), et la « dissertation" scolaire n'a plus grand chose à voir avec son ancêtre mondain (Viala, 1990).

\subsubsection{Transposition et variante scolaire : la mort de Grandet}

On peut enfin distinguer un travail transpositif, particulièrement visible dans tout ce qui concerne les définitions et les délimitations mêmes du concept « genre » à l'aide des différentes théories « savantes », et qui constituent les formes scolaires du genre ${ }^{(13)}$. On peut voir également à l'œuvre une forme de transposition dans la

(12) Le phénomène que je décris ici me semble pouvoir rendre compte de la création de la dissertation scolaire, dont Chervel $(2005$, p. 83) a montré qu'elle était liée à l'invention de l'explication de texte, « exercice annexe totalement original, et qui s'est avéré, dans les années 1880-1890, indispensable à la pratique de la dissertation littéraire ». Cf. aussi Chervel, 2006, p. 668-688.

(13) Ce travail transpositif peut s'accompagner de réécritures, et s'apparenter à la redéfinition, évoquée supra. 
scolarisation des romans de Balzac, à travers la pratique des extraits dans les manuels, qui constituent ce que j'appelle, à la suite de Balibar (1985), la « variante » scolaire du texte, élaborée pour répondre à des finalités disciplinaires. Je le montrerai ici à travers l'étude de la scène d'agonie du père Goriot, bien présente dans mon corpus de manuels ${ }^{(14)}$ (9 occurrences entre 1900 et 2004), qui met en évidence l'évolution des finalités scolaires et disciplinaires. En effet, si les extraits de Balzac ont été utilisés à des fins morales pendant une bonne moitié du $\mathrm{XX}^{\mathrm{e}}$ siècle (Denizot, 2010), cette finalité a peu à peu cohabité avec d'autres finalités, auxquelles elle a fini par laisser la place.

Je ne m'attarderai pas sur les coupes pratiquées dans les manuels à l'intérieur même du texte ${ }^{(15)}$ : il est clair qu'elles répondent à des impératifs idéologiques, d'adoucissement en quelque sorte du texte. Les manuels font des choix, parfois différents, mais il suffit de confronter la variante scolaire de Braunschvig (1921/1955) avec la version princeps ${ }^{(16)}$ pour constater l'extrême cohérence des coupes pratiquées. Ce sont tout d'abord certains passages violents qui sont supprimés, notamment ceux qui relèvent du registre corporel («Oui, je le vois, pour elles, l'habitude de m'ouvrir les entrailles a ôté du prix à tout ce que je faisais. Elles auraient demandé à me crever les yeux, je leur aurais dit “Crevez-les !” Je suis trop bête. »). Ce sont aussi les allusions à l'histoire d'amour entre Rastignac et l'une des filles de Goriot («Dites-lui que vous ne l'aimerez plus si elle ne veut pas venir. Elle vous aime tant qu'elle viendra. »); en revanche, le passage plus ambigu où Goriot, appelant Rastignac son «fils », le conjure d'aimer sa fille... « comme un père », est conservé, puisque l'amour y est ici familialisé, si l'on peut dire. Sont enfin coupées les phrases terribles où Goriot s'en prend à l'institution même du mariage, et aux gendres qui souillent les filles (« Le gendre est un scélérat qui gâte tout chez une fille, il souille tout. Plus de mariages! C'est ce qui nous enlève nos filles, et nous ne les avons plus quand nous mourons. ») : la connotation sexuelle a visiblement effrayé les auteurs du manuel.

Le découpage du texte fait apparaître des différences importantes entre les manuels avant et après 1970. Chez Balzac, l'agonie de Goriot est en effet très longue, et occupe plus de 25 pages dans une édition de poche. De nombreux extraits sont donc possibles, même si l'on veut s'en tenir à la scène la plus terrible, qui voit le père Goriot en proie au délire pendant que Rastignac veille sur lui. Or sur les neuf extraits des manuels, six proposent un découpage très semblable, d'une assez grande ampleur, avec une caractéristique intéressante, qui permet de voir à l'œuvre le souci de donner à lire une page exemplaire : dans les cinq manuels les plus anciens, les extraits se terminent sur un moment dramatique, la bénédiction du père Goriot. Ce qui a été choisi comme point final à l'extrait n'est donc pas du tout le point final de la scène, qui se poursuit avec l'entrée de l'étudiant en médecine Bianchon, ramenant la scène à des choses plus prosaïques : il tâte le pouls du bonhomme, dit qu'il faut trouver de l'argent, et va mettre en gage la montre de Rastignac. Mais l'extraction est bien ici désancrage ${ }^{(17)}$ : en coupant la scène juste au moment de la bénédiction du

(14) Pour analyser la scolarisation des romans de Balzac, j'ai dépouillé 143 manuels parus entre 1880 et 2007, de tous les niveaux du secondaire, enseignement général et professionnel.

(15) Avant 1970, seuls Chassang et Senninger (1966) laissent la scène intacte ; en revanche, après 1970, aucune coupe n'est effectuée à l'intérieur du texte.

(16) J'emprunte le terme à Renée Balibar (1985, p. 324).

(17) J'emprunte ce concept de « désancrage » à Yves Reuter (1990, p. 10) qui caractérise ainsi les opérations d'extraction et d'insertion « qui inscrivent un texte dans le champ littéraire en modifiant les pratiques qui l'ont constitué dans son espace social d'origine ». 
père, on transforme cette bénédiction en ultime parole ${ }^{(18)}$, avec une charge dramatique et pathétique énormes, qui n'est pas dans Balzac puisque l'agonie est loin d'être terminée et ne nous épargnera pas au passage quelques détails sordides. D'ailleurs, le dernier soupir de Goriot n'est un «soupir de joie », comme écrit Balzac, que par une illusion, puisque Goriot prend Rastignac et Bianchon pour ses filles, et meurt en leur serrant la main. On n'est pas loin chez Balzac d'une forme d'ironie tragique, qui n'a plus grand chose à voir avec le pathos de la bénédiction que privilégient les manuels.

Un même découpage peut également produire des effets de lecture différents selon l'ancrage de l'extrait, et notamment son titre et son paratexte. Chassang et Senninger (1966), qui gardent quasiment intacts les frontières traditionnelles de l'extrait, parasitent totalement sa dimension édifiante en lui donnant un titre très distancié, "Le goût de la scène à effet » : il s'agit nettement dans ce manuel de viser aussi des savoirs savants sur Balzac - à côté ou en plus des objectifs moraux. Cela dit, le fait qu'ils s'inscrivent dans un découpage scolaire déjà traditionnel n'est pas sans poser question : faut-il y voir une forme d'inertie, les manuels se copiant les uns les autres dans une longue chaîne qui finit par former une certaine « culture scolaire " ${ }^{(19)}$ ? Est-ce au contraire une volonté délibérée de jouer de cette «culture scolaire " pour viser d'autres objectifs, toute nouveauté scolaire s'imposant plus facilement lorsqu' elle vient s'inscrire dans ce qui est déjà ? Il n'est pas simple de trancher, et sans doute les deux positions coexistent-elles et se superposent-elles plus souvent qu'elles s'excluent. On le voit aussi dans un manuel contemporain (Lancrey-Javal, dir., 2004), qui choisit un extrait de cet extrait, si je puis dire ${ }^{(20)}$, pour un exercice sur les personnages et les registres : est-ce le poids de la tradition scolaire ou bien s'agit-il ainsi de rassurer les enseignants, en reprenant un passage connu pour travailler sur de nouveaux objets (les registres, apparus dans le programme de 2000) ? Là aussi, il est difficile de répondre. Mais quoi qu'il en soit, il me semble que cette ambivalence apparaît constamment dès lors qu'on étudie de près les savoirs scolaires.

\subsection{Scolarisation et secondarisation : les genres scripturaux scolaires}

La scolarisation de certains genres est souvent articulée avec ce que je nomme la secondarisation, terme que j'utilise pour décrire la transformation de genres scolaires et/ou extrascolaires déjà existants en nouveaux objets disciplinaires ${ }^{(21)}$. J'emprunte à Bakhtine (1979/1984) son opposition célèbre entre genres premiers et gen-

(18) Le choix de Mitterand (1986) est à cet égard intéressant, puisqu'il rompt - visiblement délibérément - avec cette tradition : les frontières de l'extrait sont globalement les mêmes, mais il se termine juste avant la bénédiction, sur les mains de Goriot qui s'agitent comme pour saisir les cheveux de ses filles. Ce découpage donne à lire le passage autrement, en laissant une impression d'inachevé, de suspension : Goriot est mourant, mais pas encore mort. Et surtout, la suppression de la bénédiction finale produit un effet nettement moins édifiant.

(19) Sur cette notion de «culture scolaire», voir notamment Chervel (1998) et plus récemment Jacquet-Francillon et Kambouchner (2005).

(20) Le manuel ne retient en effet qu'une petite partie de la scène.

(21) J'utilise le terme dans un sens un peu différent d'Élisabeth Bautier et Roland Goigoux (2004), qui désignent ainsi le « rapport second au savoir » que l'école demande aux élèves, à savoir une décontextualisation avec changement de finalité. Jean-Paul Bernié, Martine Jaubert et Maryse Rebière (2008) reprennent l'expression dans un sens assez voisin, pour désigner le processus de mise à distance des pratiques langagières ordinaires dans le cadre de la «communauté discursive» qu'est la classe. Ce ne sont pas, pour ma part, les processus 
res seconds, mais en la reformulant : en effet, ce n'est pas cette opposition en tant que telle qui m'intéresse, mais la propriété que Bakhtine attribue aux genres seconds, celle de pouvoir « absorber et transmuter » les genres premiers, dans une opération quasi alchimique. Je reformule cette propriété de cette façon : les genres «absorbent et transmutent " sans cesse d'autres genres, et ces genres transmutés peuvent «perdre leur rapport immédiat au réel existant », c'est-à-dire aux conditions socio-historiques et/ou énonciatives qui ont vu naître les genres d'origine. Dans cette reformulation, "premiers » et «seconds » ne définissent pas des catégories fermées de genres, mais un processus, puisqu'un genre « second » peut devenir " premier » et être à son tour absorbé et transmuté. Cette secondarisation conduit donc à la création de nouveaux genres à partir de genres "premiers » déjà existants, par une sorte de «dérivation », si l'on peut dire : on transforme et adapte un genre déjà existant (le portrait, le discours, la dissertation) en un genre disciplinaire autonome avec son fonctionnement et ses finalités propres ${ }^{(22)}$. La discipline français a ainsi inventé des genres disciplinaires spécifiques, à usage scriptural comme ceux que je viens de citer, mais aussi à usage lectoral comme les textes fondateurs (Denizot, 2009a) ou le « biographique », genre éphémère des programmes de lycée (Denizot, 2006b et infra).

Je vais m'arrêter ici sur les genres scolaires scripturaux issus des pratiques rhétoriques qui dominent l'enseignement au $\mathrm{XIX}^{\mathrm{e}}$ siècle, marqué par les humanités, et qui voient dans les genres des objets scolaires tournés essentiellement vers des pratiques scripturales. Je ne m'attarderai pas sur l'histoire scolaire de la rhétorique, bien balisée par les travaux de Françoise Douay-Soublin (notamment 1999) mais sur une catégorie qui, sans appartenir en tant que telle aux grandes catégories rhétoriques, n'en a pas moins une existence et une importance réelle : il s'agit des " genres » que sont la narration, la description, le tableau, etc. Ces genres ont longtemps organisé les manuels, en commençant notamment par le Noël et Delaplace, l'un des plus célèbres recueils du XIX ${ }^{\mathrm{e}}$ siècle ${ }^{(23)}$, dont la destination première, comme l'a montré Douay-Soublin (1997, p. 166), est la formation rhétorique.

Les deux tomes du recueil (prose et poésie) sont organisés de la même manière, autour des mêmes entrées : " narrations », " tableaux ", " descriptions ", " définitions », « fables et allégories », « philosophie morale et pratique », « lettres », « discours et morceaux oratoires », " dialogues », et enfin " caractères et portraits, et parallèles ». Or ces catégories empruntent, pour la plupart d'entre elles, leur dénomination à différentes parties du système rhétorique (invention, disposition et élocution), mais restent hétérogènes et hétéroclites. Les auteurs de travaux sur la question utilisent d'ailleurs tous un terme différent pour les qualifier : " genre ", " types de textes et sujets », « rubriques », « subdivisions » de l'art d'écrire, « topiques »; quant à Noël et Delaplace, il s'en tiennent dans leur préface au terme de « mor-

de mise à distance qui m'intéressent ici, mais plutôt les processus de recomposition et reconfiguration. Dans ce sens, mon emploi du terme s'apparente davantage à celui de Bernard Schneuwly (2007a), qui l'utilise pour décrire les progressions curriculaires en matière de genres textuels.

(22) L'idée de la secondarisation me paraît préférable à la distinction opérée par Schneuwly (2007b) entre genres scolaires (la description, la narration, la dissertation) et genres scolarisés (adaptations et transformations des genres existants) : il me semble que les « genres scolaires » sont d'anciens genres scolarisés, lesquels tendent à devenir « scolaires » et/ou à être à nouveau scolarisés. Plutôt que de parler du «mélange extrêmement complexe de genres scolaires/scolarisés» (id., p. 18), je préfère parler d'un processus toujours susceptible de se mettre en marche, qui brouille sans cesse la frontière entre «scolaire » et «scolarisé ».

(23) La BNF recense 29 éditions entre 1804 - date de la première édition - et 1862 . J'étudie la deuxième édition, de 1805 . 
ceaux ». Cette dénomination est d'ailleurs conforme à la tradition néo-rhétorique, qui met à l'honneur la declamatio, c'est-à-dire l'improvisation réglée sur un thème, et qui donne au « morceau» (paysage, portrait, etc.) une existence autonome, ce qui lui permet de quitter le discours oratoire et de s'intégrer dans la narration (Barthes, 1970/2002, p. 542-543).

Ces « morceaux de composition », qui appartiennent à la tradition des progymnasmata de l'enseignement antique, constituent ainsi des genres scolaires, que je décris donc comme résultant d'un processus de secondarisation : c'est par absorption et transmutation de catégories hétérogènes, empruntées à la rhétorique, que l'école crée ces nouveaux genres, qui perdent en grande partie le rapport avec la réalité qui les a vus naître, et qui acquièrent ainsi une existence autonome, propre à l'école. Ils disparaissent d'ailleurs presque entièrement en tant que genres littéraires lorsque la littérature devient un corpus de textes à lire, à expliquer et à admirer ${ }^{(24)}$. Mais, en tant que genres scolaires, ils survivent à travers deux pratiques disciplinaires: tout d'abord celle des extraits et des morceaux choisis ${ }^{(25)}$, qui les donnent à lire à différentes époques; et surtout via les pratiques d'écriture scolaire, puisque c'est la composition française puis la rédaction et plus récemment l'écriture d'invention qui prolongent en partie l'enseignement de la rhétorique, disparu en tant que tel au début du XX $X^{\mathrm{e}}$ siècle (Petitjean, 2003).

Mais si les genres hérités de la rhétorique subsistent dans les pratiques d'écriture disciplinaires, c'est au prix d'un désancrage qui peut apparaître comme paradoxal : plus les portraits, descriptions et autres récits deviennent des exercices scolaires, moins leur appartenance à des genres littéraires et textuels (ici rhétoriques) est lisible et revendiquée. Ce paradoxe n'est qu' apparent, et s'explique par le processus de secondarisation qui en fait des objets scolaires autonomes, au point d'ailleurs d'influencer parfois à leur tour la culture de la société globale. Claude Abastado (1981) a par exemple montré, à partir d'une étude de faits divers dans différents journaux, que les modèles scolaires hérités de la composition française sont flagrants dans les textes de presse, pour des raisons qui tiennent à l'efficacité recherchée. On pourrait faire sans doute la même remarque à propos du modèle de la dissertation scolaire, qui informe bon nombre d'écrits sociaux, et étudier les influences des productions et des genres scolaires sur les genres sociaux.

(24) Certains manuels conservent encore ce classement par genres, et ce caractère atypique est intéressant pour voir à l'œuvre les démarches de transition classiques à l'école. C'est ainsi que le manuel de Julien Boitel (1901) propose des Morceaux choisis classés selon les genres littéraires, mais ce qu'il nomme « littéraire », ce n'est pas ce qu'on nomme ainsi déjà à cette époque (roman, poésie, etc.), mais bien les genres scripturaux que sont la narration, la description, etc. Boitel s'en justifie d'ailleurs longuement dans sa préface, avec des arguments justement d'ordre pédagogique : il s'agit encore de proposer aux maîtres et aux élèves des modèles d'écriture.

(25) La plupart des grandes anthologies de la fin du XIX ${ }^{\mathrm{e}}$ et du début du XX ${ }^{\mathrm{e}}$ siècle adoptent un ordre chronologique, puis historique, plus conforme aux nouvelles exigences de l'enseignement de 1'histoire littéraire et, dans une moindre mesure, de 1'explication de textes. Mais le découpage par «genres » ne disparaît pas pour autant, et on le voit subsister et rester encore lisible à travers les titres des extraits, comme le signale ainsi explicitement Marcou, auteur de plusieurs volumes de morceaux choisis souvent réédités, dans une de ses préfaces (1884, p. V; c’est moi qui souligne) : «Sans méconnaître les raisons et les avantages du plan qui consiste à classer les morceaux choisis par "genres", nous avons cru préférable celui que nous avons choisi. Il est toujours facile de reconstituer le classement par genres ; le titre seul des morceaux y aide déjà : il le serait beaucoup moins de rétablir la suite de notre histoire littéraire en replaçant dans l'ordre des temps les passages distraits de cet ordre naturel pour entrer dans un plan conventionnel. » 


\subsection{Scolarisation et reconfiguration : l'exemple du «biographique »}

La reconfiguration est un concept souvent employé actuellement dans les sciences humaines et qui emprunte au domaine de l'informatique une métaphore venue de la géographie (la configuration d'un lieu), permettant de décrire le caractère malléable de certains objets : configurer ou reconfigurer un ordinateur, ce n'est pas seulement installer du matériel et des logiciels, c'est agencer et éventuellement personnaliser l'ensemble, de façon plus ou moins originale, selon les besoins et/ou les préférences de l'utilisateur. Les processus de reconfiguration qui m'intéressent sont ceux qui recomposent et réorganisent des genres littéraires et textuels pour leur donner de nouvelles formes et de nouveaux enjeux. Ce sont des processus, c'est-àdire qu'il faut envisager la reconfiguration comme susceptible de n'être jamais complètement stable ni achevée : tel genre peut ainsi être reconfiguré plusieurs fois, dans diverses configurations disciplinaires. Les reconfigurations peuvent souvent donner lieu à de véritables créations, la discipline élaborant des genres qui lui sont propres et qui servent ses finalités d'enseignement et d'apprentissage : le biographique au lycée ( $c f$. Denizot, 2006b) et les textes dits «fondateurs " au collège en sont des exemples récents; le « roman réaliste» ( $c f$. Denizot, 2010) et la «tragédie classique » ont quant à eux déjà été reconfigurés à plusieurs reprises.

J'étudierai ici la reconfiguration opérée récemment par les programmes de lycée, qui inventent un nouveau genre, le biographique, d'ailleurs éphémère : apparu pour la première fois sous ce nom dans les programmes de première de 2001, il en a disparu lors du remaniement du programme en vigueur à la rentrée 2007 : seule demeure l'autobiographie (pour les classes de $1^{\text {re }} \mathrm{L}$ ), genre davantage stabilisé. C'est cet échec qui m'intéresse ici.

Le biographique, tel qu'il est institué par le programme de 2001 (« récits de vie, mémoires, journal intime, biographie, autobiographie, roman autobiographique »), est une catégorie ambivalente, écartelée entre genres biographiques et autobiographiques et entre fiction et réalité. Pour comprendre comment les manuels et les enseignants se sont emparé de cette nouvelle catégorie générique, j'ai analysé 13 manuels de littérature de première (c'est-à-dire la quasi-totalité des manuels parus entre 2001 et 2005), et mené une enquête par questionnaires ${ }^{(26)}$ auprès de 30 enseignants de première, notamment sur les textes lus et étudiés en classe, sur les problématiques choisies ainsi que sur la définition et les notions utilisées avec les élèves. Les résultats sont assez concordants. L'autobiographie se taille la part du lion, autour de quelques œuvres canoniques et incontestées (Les Confessions de Rousseau, Les Essais de Montaigne et les Mémoires d'Outre-Tombe de Chateaubriand), qui constituent le fonds du corpus autobiographique, et autour de nouveaux classiques (particulièrement Sartre, Cohen, Ernaux, Juliet, Perec et Sarraute). Ces phénomènes de classicisation vont d'ailleurs de pair, comme souvent à l'école, avec la constitution d'un corpus de morceaux choisis, notamment les pactes autobiographiques (sur 107 extraits étudiés, 20 sont des pactes, et le «préambule» de Rousseau est étudié par 10 enseignants sur 30, et présent au titre de lecture complémentaire dans 16 descriptifs sur 35): ce n'est pas tant la «belle page» qui est ainsi sélectionnée que la page qui permet un usage directement en prise avec les savoirs disciplinaires autour de l'autobiographie. Les finalités disciplinaires priment ici sur les finalités esthétiques et littéraires. D'ailleurs, l'élargissement du corpus autobiographique s'ac-

(26) 24 réponses sont accompagnées d'un ou plusieurs descriptifs (de 1'année en cours et de l'année précédente) pour l'épreuve anticipée de français (EAF), qui sont une sorte de vitrine du travail effectué en classe. 
compagne d'une recatégorisation de certaines œuvres : c'est le cas notamment des Essais, présents dans les manuels avant 2001 mais selon d'autres points de vue (l'humanisme par exemple), et dont la relecture autobiographique oblige à ne sélectionner que les passages qui peuvent correspondre à la définition actuelle de l'autobiographique. Or cette définition est inspirée en grande partie des travaux de Philippe Lejeune, pour lesquels le modèle est nettement Rousseau et non Montaigne, créateur de l' " essai », genre moins facilement modélisable.

La place des autres genres du biographique est plus contrastée : le journal intime, protéiforme et difficilement réductible en extraits, reste très marginal dans les manuels et chez les enseignants (seulement 6 enseignants sur 30 déclarent avoir étudié «très souvent » ou « assez souvent » le journal depuis 2001). Quant à la biographie, elle fait une entrée spectaculaire dans les manuels et, loin d'être cantonnée comme auparavant aux notices biographiques, elle devient véritable objet disciplinaire à part entière. Mais cette légitimation est corrélée à une littérarisation : d'une part ce sont les biographies d'écrivains qui sont privilégiées, et d'autre part ce sont souvent des auteurs prestigieux ou reconnus qui sont convoqués (c'est ainsi par exemple que La vie de Rancé, de Chateaubriand, absent des manuels avant 2001, est présent dans 6 des 13 manuels de mon corpus). La version scolaire du genre biographique est devenue nettement littéraire, au double sens du terme d'ailleurs, en tant que le littéraire est une propriété textuelle (la littérarité) mais aussi un thème (les biographies d'écrivains). Chez les enseignants enquêtés, la place de la biographie est également sans précédent, mais elle est généralement articulée avec l'autobiographie, en tant que texte « complémentaire »: sa place reste celle d'un genre ancillaire, qui n'existe qu'en complément d'un genre plus noble. Non seulement elle n'est pas suffisamment légitime pour faire l'objet de lectures analytiques, mais dans la plupart des cas, elle a besoin de l'autobiographie pour justifier en quelque sorte sa présence.

Deux genres enfin, le roman biographique et autobiographique, ont une place particulière, en ce qu'ils appartiennent au domaine de la fiction. Dans les manuels comme chez les enseignants enquêtés, ils sont généralement articulés aux genres non-fictionnels correspondants. Le roman autobiographique, bien présent dans les manuels et dans l'enquête (15 enseignants sur 30 déclarent l'avoir étudié « très »ou " assez souvent»), permet de faire réfléchir sur l'autobiographie (je reviens infra sur la question, à propos de l'amphitextualité). Quant au roman biographique, il est en fait assez peu présent dans les manuels, qui convoquent plutôt des biographies romancées pour questionner les limites du genre de la biographie, genre hybride, entre hagiographie, histoire et roman; les enseignants enquêtés choisissent quant à eux des œuvres à la frontière de la biographie et de l'autobiographie, comme certains textes d'Annie Ernaux, qu'ils étudient dans le cadre de séquences consacrées à l'autobiographie, et non à la biographie. On retrouve ici le statut ancillaire de la biographie déjà évoqué précédemment.

L'introduction du biographique dans les programmes a donc mis sous le projecteur des genres jusqu'ici ignorés ou méprisés et ouvert le corpus scolaire des textes au lycée, soit par l'introduction de nouveaux auteurs, soit par la découverte ou la redécouverte, chez des auteurs reconnus, d'œuvres moins connues ou moins scolarisées. Cet élargissement du corpus des textes du lycée a débouché aussi sur une recatégorisation générique de certains textes. Mais tout cela n'aura pas suffi à stabiliser le « biographique » et la domination d'une partie du corpus (l'autobiographie notamment) au détriment d'une autre partie pourtant constitutive du genre scolaire (le journal intime par exemple) peut également expliquer le resserrement du programme de 2007 autour de l'autobiographie.

Cette étude du biographique met ainsi en évidence un phénomène qui me semble 
être un fonctionnement disciplinaire récurrent, et qui se présente sous une forme paradoxale. Pour qu'un nouvel objet s'ancre dans l'école, il lui faut tout d'abord pouvoir s'inscrire dans une forme de tradition scolaire. C'est ainsi que l'autobiographie est devenue un objet de travail ces dernières années, en s'inscrivant dans des pratiques de lecture littéraire qui valorisent les « grands » auteurs et les œuvres patrimoniales ; c'est ainsi également que la biographie peut devenir un objet de travail si elle se cantonne aux biographies littéraires. Mais il faut en même temps que le nouvel objet puisse résister à une forme d'assimilation par cette même tradition, faute de quoi il est plus ou moins rapidement absorbé, réduit au sens quasi chimique du terme. On a vu ainsi le biographique se réduire à sa composante autobiographique, et les genres biographiques considérés comme mineurs (biographie, journal) disparaître.

\section{Amphitextualité : un concept pour analyser les solidarités textuelles}

Il convient de faire une place à part au concept d'amphitextualité qui vise à décrire les solidarités textuelles dans lesquelles sont pris les textes et les genres. Cette question des solidarités disciplinaires me semble en effet être une problématique essentielle à l'école, la forme scolaire (au sens de Vincent, 1980) tendant à procéder par découpage (des heures de cours, des programmes, des disciplines) et à poser les objets scolaires les uns à côté des autres, parfois de manière réfléchie (dans le cadre des progressions par exemple, qui peuvent également être un principe d'organisation des manuels), parfois de manière plus aléatoire (ne serait-ce que parce que les progressions sont rarement complètement cohérentes, et présentent généralement des discontinuités, ou, pour reprendre l'exemple des manuels, parce que tous les textes ne peuvent y être articulés entre eux). Je décris donc ces solidarités textuelles comme un autre mode de rapport possible des textes entre eux, à côté des cinq types de relations transtextuelles définies par Genette (1982, p. 11-12) : métatextualité, hypertextualité, paratextualité, intertextualité et architextualité. À ces types de relations, j'ajoute celle qui relie un texte à un ou plusieurs textes à côté desquels il est posé, relation qui n'est pas purement paratextuelle (puisque chacun des textes peut aussi être accompagné d'un paratexte), mais qui est d'ordre contextuel et que je nomme, sur le même modèle et pour désigner spécifiquement ce type de « contextualité » : l'amphitextualité (du grec ancien amphi: autour de, des deux côtés) ${ }^{(27)}$.

Or, dans le cas des genres textuels, ces phénomènes d'amphitextualité sont importants, parce que les textes et les genres, rarement isolés, sont ainsi pris dans des réseaux qui peuvent modifier le point de vue que l'on a sur eux. Je l'ai montré notamment à travers les classements dans les CDI ${ }^{(28)}$, qui donnent à voir ou non certains genres en fonction de leur place dans le classement, et donc en fonction de ce qui les accompagne : certains documentalistes classent par exemple les autobiographies avec les journaux intimes, les mémoires et les souvenirs (en 848 de la classification Dewey: «Littérature française/Écrits divers »), recomposant ainsi une sorte de catégorie écrits intimes (assez proche du biographique tel qu'il apparaissait dans les programmes du lycée des années 2000); d'autres rangent les autobiographies en histoire dans la classe 920 («Histoire/Biographie, généalogie »), et donc avec les biographies; d'autres encore (les plus nombreux) en 800, avec les romans. Or, selon

(27) La proximité sémantique avec les «amphis » de l'université ou du lycée ajoute au terme une connotation scolaire qui n'est pas déplacée.

(28) Pour une première version de mon étude sur les CDI, $c f$. Denizot, 2005. 
les choix opérés, l'autobiographie est ou non visible comme genre spécifique, et est ou non perçue comme genre littéraire. Les biographies posent également des problèmes de classement, mais leur statut est encore plus incertain, et leur appartenance à la littérature encore plus conditionnelle : dans certains CDI, les biographies sont partagées entre l'histoire (en 920), la littérature (800) et les différents domaines correspondant au biographié - en 500 ( «sciences ») pour une biographie d'Einstein, en 700 (« arts ») pour une biographie de Van Gogh, etc. - ; quant aux biographies rangées en 800, elles peuvent être soit avec les romans, soit en histoire littéraire, soit à proximité de l'œuvre de l'écrivain biographié : l'appartenance au domaine littéraire tient alors au sujet traité, et non à la réception de l'œuvre ni au jugement esthétique qui pourrait être porté sur elle.

Ce concept d'amphitextualité permet surtout d'approcher la construction des genres littéraires dans les manuels, pour comprendre comment sont construits et reconfigurés les genres selon les usages et les configurations disciplinaires. On en a vu un premier exemple supra avec la tragédie classique : prise dans des réseaux amphitextuels axés sur la rhétorique, l'histoire littéraire, les formes dramatiques ou les autres genres tragiques, la tragédie classique est définie et délimitée chaque fois de manière sensiblement différente, au point que ce genre apparemment immuable se révèle finalement d'une réelle instabilité disciplinaire. Je m'attarderai ici sur deux exemples : les amphitextes des extraits de Balzac dans les manuels scolaires, qui dessinent par exemple tantôt un Balzac représentant du genre « roman réaliste », tantôt un Balzac appartenant au courant romantique, parfois même autour d'un même extrait ${ }^{(29)}$; et l'amphitextualité du roman autobiographique dans les manuels scolaires avant et après 2001, qui modifie sensiblement les enjeux du genre.

\subsection{Le corpus balzacien : vulgates scolaires et amphitextualités}

Pendant une grande partie du XX $\mathrm{XX}^{\mathrm{e}}$ siècle, les manuels et les histoires littéraires réduisent Balzac à son " réalisme ». Les manuels après 1970 sont généralement plus nuancés, et prennent acte des liens qui unissent Balzac et le romantisme, soit explicitement, soit plus implicitement, en mettant notamment en avant certains textes relevant du fantastique voire de l'occultisme. Mais il semble que subsiste une sorte de vulgate scolaire, pour laquelle les romans de Balzac sont des représentants prototypiques du roman réaliste du XIX ${ }^{\mathrm{e}}$ siècle, aux côtés notamment de Flaubert et Zola, et qui définit le réalisme comme un genre romanesque et non comme la « catégorie esthétique » (Dufour, 1998, p. 7) qu'y voient les critiques modernes. L'existence de cette vulgate scolaire est visible à travers deux exemples, pris cette fois non plus dans les manuels, mais dans les listes du baccalauréat des années 1990, étudiées par Bernard Veck, et dans des ouvrages parascolaires.

Veck (1992), dans sa recherche sur les listes d'oral à l'EAF, montre comment les enseignants, pour délimiter un corpus qu'ils considèrent comme représentatif des genres, procèdent par des élections et des exclusions qui construisent une version scolaire du genre. Or le " roman scolaire », explique-t-il, est généralement réduit au roman réaliste du XIX ${ }^{\mathrm{e}}$ siècle (Balzac, Stendhal, Flaubert et Zola essentiellement), excluant de ce fait des romanciers comme Hugo, largement présent par sa poésie et son théâtre, mais quasi absent en ce qui concerne ses romans (p. 222 ; c'est moi qui souligne) :

Le roman hugolien n'entre pas dans la définition du genre qui caractérise le savoir scolaire, ou du moins pas au même titre que les romans de Balzac par exemple. Le

(29) J'ai consacré deux articles à la scolarisation de Balzac : cf. Denizot, 2009b et 2010. 
roman, dans sa définition scolaire, est non seulement identifié au roman du XIX siècle, mais encore à ce que l'on peut appeler, globalement et imparfaitement, en se servant d'une étiquette scolaire justement, le roman réaliste.

Le «genre scolaire » roman étant défini selon Veck par un double découpage, dans l'ensemble des auteurs puis dans l'histoire du genre, c'est ce découpage qui en quelque sorte « transpose » le genre ${ }^{(30)}$. Or, dans ce roman scolaire, Balzac est associé à des romanciers considérés également comme « réalistes », et non aux autres romanciers romantiques, d'ailleurs quasiment absents des listes.

Cela se retrouve dans les manuels et les parascolaires, et ce sont les amphitextualités dans lesquelles sont pris les extraits des romans balzaciens qui leur donnent un sens, et parfois d'ailleurs les enferment : associés à Flaubert et à Zola plus qu'à Dumas et Mérimée, les romans de Balzac illustrent le " genre réaliste ", parfois jusqu'à l'absurde. C'est ainsi que, dans un ouvrage de 1992 consacré au " commentaire composé » (Anglard, 1992, p. 170 sqq. ), la fameuse description au début du Lys dans la vallée ouvre la section «La description réaliste », puis est suivie d'un extrait de Flaubert et d'un de Zola. Mais le corrigé, entièrement rédigé et organisé autour de « la situation de communication » (le point de vue de Félix et la fonction explicative), «l'échange des qualités » (les correspondances entre la vallée et la femme aimée), et « la transfiguration amoureuse », avant de conclure explicitement sur l'échec du réalisme balzacien, illustre surtout l'impasse à laquelle conduit cette catégorisation trop applicationniste.

La vision de Balzac comme précurseur du naturalisme offre ainsi un cadre d'analyse un peu réducteur. Cela est sans doute d'autant plus apparent que, lorsque de nouvelles finalités disciplinaires apparaissent, une nouvelle amphitextualité se met en place. Dans les années 1990, par exemple, les textes officiels prévoient des programmes d'œuvres en classe de première pour la dissertation, et proposent en 1995 " un roman d'apprentissage d'un auteur de langue française du XIX ${ }^{\mathrm{e}}$ siècle ». Certains romans de Balzac, et notamment bien sûr Le Père Goriot, sont alors mis en avant, aux côtés des romans de Stendhal, de L'Éducation sentimentale de Flaubert ou de Bel-Ami de Maupassant, mais pas avec les romans de Zola. Cette nouvelle configuration générique met au centre non plus le « réalisme » balzacien, mais le personnage, et s'inscrit ainsi dans une tendance qui privilégie en particulier Vautrin, personnage éminemment romanesque. Quant à la configuration actuelle, elle fait bouger l'image scolaire de Balzac, notamment sous l'influence des programmes des années 2000 : « un mouvement littéraire et culturel » y figure au programme de la classe de seconde, et de nombreux enseignants choisissent le romantisme. Or, les programmes imposant la lecture pour cet objet d'étude d'au moins une œuvre intégrale, certains romans de Balzac sont étudiés dans ce cadre : le site Weblettres ${ }^{(31)}$ par exemple propose dans une synthèse intitulée «le Romantisme : idées de lectures cursives ", une liste d'ouvrages parmi lesquels figurent dix romans de Balzac. Ce rapprochement entre Balzac et les écrivains romantiques contribue ainsi à rendre vi-

(30) Veck analyse en effet ce « genre scolaire » à l'aide du modèle de la transposition didactique hérité de Chevallard. Il me semble pourtant que c'est justement parce que l'on ne peut nommer le roman «scolaire » autrement que par l'étiquette «scolaire » que l'on assiste ici plus à une construction qu'à une transposition : classer et étiqueter sont des opérations intellectuelles et culturelles, et l'étiquette ne préexiste pas "naturellement» au classement. Si seule une étiquette scolaire permet de classer un certain nombre d'œuvres, c'est que le découpage opéré fait sens dans l'univers scolaire, voire ne fait sens que dans 1'univers scolaire. Autrement dit, l'école ne transpose pas ici un savoir savant qui serait le genre du « roman réaliste du XIX ${ }^{\mathrm{e}}$ siècle », elle 1'invente.

(31) http ://www.weblettres.net/spip/article.php3 ?id_article=29 [consulté 1e 2 mars 2008]. 
sible un autre Balzac, et à introduire dans le corpus scolaire d'autres œuvres de Balzac.

Pris dans d'autres réseaux de solidarités amphitextuelles, les extraits de Balzac sont donc lus autrement, et dans un ouvrage récent (Hubert, 2003) consacré au romantisme (à destination des classes de seconde), la description de la vallée du Lys dans la vallée qui était choisie comme modèle de description réaliste devient cette fois une description emblématique du romantisme, aux côtés d'autres auteurs romantiques. Une consigne appelle à « interpréte[r] les éléments lyriques du récit », un plan de commentaire propose d' " analyser le cadre naturel », puis de « souligner les correspondances entre la femme aimée et le cadre dans laquelle elle vit », pour « conclure brièvement par une analyse de la vision du monde qui se dégage de ce regard balzacien ». Il est notable que le terme « réaliste » n'y apparaît jamais. Il est surtout évident que l'analyse gagne en cohérence, et permet de dépasser la fausse évidence du « récit réaliste».

\subsection{Les amphitextualités du roman autobiographique}

L'appellation « roman autobiographique» se trouve dans les anthologies d'avant 2001 pour des œuvres comme $\dot{A}$ la recherche $d u$ Temps perdu, ou pour la trilogie de Jules Vallès. Le roman autobiographique est ainsi depuis un certain temps identifié par l'école, mais elle n'en fait pas nécessairement un genre à part : c'est une sous-catégorie du roman, tout simplement, qui renvoie essentiellement à la vie de l'auteur. Après 2001, il permet surtout de réfléchir sur l'autobiographie, à laquelle il est confronté.

On peut par exemple comparer la présentation d'un même extrait d'Adolphe de Constant dans deux manuels dirigés chez Bordas par la même équipe (Aviérinos et Prat, 1997 ; Aviérinos, Prat et Labouret, 2001). Dans le premier, une notice biographique chapeaute la page, évoque « la passion houleuse » de Constant pour Mme de Staël (dont un extrait de Corinne figure en vis-à-vis sur la page d'à côté) et se termine ainsi : «Au-delà de la confession personnelle, ce livre est l'un des premiers grands romans français à témoigner du "mal du siècle" ». L'accent est donc mis sur la «confession » de l'auteur, sa personnalité torturée, ainsi que sur l'inscription du roman dans la période romantique, évoquée ici par l'expression emblématique du « mal du siècle». En 2001, le manuel présente le même extrait, mais sans notice biographique. L'introduction du passage commence ainsi : «Sous les traits d'Adolphe, l'auteur relate sa liaison orageuse avec Germaine de Staël, tout en fusionnant le souvenir de plusieurs autres liaisons pour recomposer l'histoire d'Adolphe et d'Ellénore. » Sur la page d'à côté figure cette fois un extrait du Cahier rouge, autobiographie de Constant restée à l'état d'ébauche. L'extrait sert donc cette fois à réfléchir sur les liens entre autobiographie et roman autobiographique, ainsi que sur les processus de création, et la solidarité avec une œuvre purement autobiographique renforce nettement la catégorisation d'Adolphe en "roman autobiographique ".

Les romans autobiographiques, bien présents depuis 2001 dans les chapitres des manuels concernant les genres biographiques et/ou autobiographiques, le sont donc en solidarité avec l'autobiographie, et non plus avec le roman, et cette nouvelle amphitextualité renouvelle le regard que l'on porte sur ces textes en même temps qu'elle leur donne une nouvelle légitimité. On peut noter ainsi le retour ${ }^{(32)}$ assez re-

(32) Cette remarque se fonde sur la confrontation que je fais entre les éditions successives des manuels d'une même collection : dans les manuels des années 1990, il n'y a pas d'extrait de Colette. Elle réapparaît à l'occasion des programmes de 2001. 
marquable de Colette à la fois dans les manuels et dans les auteurs cités par les enseignants enquêtés ${ }^{(33)}$, ou la nouvelle attention portée par exemple à La Recherche $d u$ temps perdu, de Proust (présent dans 8 manuels sur 13), dont la présence dans les manuels précédents était loin d'être répandue.

\subsection{Amphitextualités aléatoires}

Dans tous ces cas, l'amphitextualité est un phénomène contrôlé, même s'il n'est pas nécessairement explicité. Mais il serait sans doute possible de prendre en compte des amphitextualités plus aléatoires, notamment dans les manuels, pour essayer d'en comprendre les effets : si l'on s'en tient aux genres, il est clair par exemple que les «mauvais genres » ${ }^{(34)}$ sont banalisés et rendus plus légitimes par leur présence aux côtés de genres plus "nobles »; et l'on peut se demander également quelle représentation du théâtre se construit pour les élèves, dans des chapitres de manuels qui ne lui sont pas spécifiquement consacrés, à travers des réseaux textuels qui privilégient généralement les genres narratifs : poser une tirade au milieu d'extraits de roman peut rendre invisible la spécificité du texte théâtral, si cette amphitextualité n'est pas un objet du travail ${ }^{(35)}$. Mais ce concept peut aussi permettre d'interroger plus largement un certain nombre d'objets disciplinaires, voire scolaires, si l'on s'intéresse aux solidarités « amphitextuelles » ${ }^{(36)}$ de certains exercices et/ou pratiques : pour en rester à la discipline français, Bertrand Daunay (2004) a montré par exemple qu'en 1969, lorsque sont définis de nouveaux exercices au baccalauréat, des solidarités unissent les deux exercices métatextuels que sont alors le commentaire et le résumé de texte ; elles disparaissent lorsque de nouvelles solidarités s'installent, cette fois entre le commentaire et l'explication de textes; or cette nouvelle amphitextualité du commentaire accompagne une évolution et une redéfinition de l'exercice, qui vise désormais à évaluer un rapport au texte littéraire. Il serait intéressant de se demander de la même façon comment l'amphitextualité des exercices accompagne voire influence les redéfinitions et les reconfigurations des exercices et des pratiques scolaires. C'est une piste à explorer, qui pourrait fournir une entrée pour travailler plus spécifiquement la question des exercices et des consignes liés aux genres.

\subsection{Point de vue, visibilité et invisibilité}

Dans le cas des genres littéraires et textuels, l'amphitextualité fait apparaître assez nettement un phénomène récurrent : nommer, définir et délimiter un genre, c'est tout d'abord poser un certain regard sur un texte. D'une certaine manière, les genres sont affaire de point de vue. Tel texte, selon le point de vue que l'on adopte, peut appartenir à tel ou tel genre, et tel genre, selon le point de vue que l'on adopte, peut être décrit comme tel autre genre. On l'a vu par exemple à propos de certaines œuvres et certains genres biographiques, comme le roman autobiographique par exemple, qui a pu, selon les finalités disciplinaires, être considéré comme relevant du genre roman ou du genre biographique. Cela est sans doute valable d'ailleurs pour un grand

(33) Sido est citée par six enseignants, ce qui la place dans le trio de tête pour les extraits étudiés, aux côtés des Confessions de Rousseau (25 extraits) et des Mémoires d'outre tombe (9 extraits), et à égalité avec Enfance de Sarraute.

(34) Je reprends ici le titre d'un ancien numéro de Pratiques (1987) consacré à la paralittérature.

(35) Par exemple dans les activités de «tri de textes ».

(36) Dans un sens imagé, puisqu'il faut alors étendre le concept à autre chose que des «textes » - extension qui ne va pas de soi et qu'il faudrait donc mettre à l'épreuve. 
nombre de notions (et pas seulement scolaires), qui prennent différentes formes selon la manière dont on les construit. Mais on peut rapprocher cette question du point de vue de ce que j'appelle la visibilité et l'invisibilité des genres : non seulement les genres - qu'ils soient littéraires, textuels, disciplinaires, etc. - sont des objets socio-historiques - et donc instables - mais le fonctionnement des objets scolaires fait que certains genres sont à certaines périodes invisibles à l'école, et que cette invisibilité ne va pas forcément de pair avec leur visibilité extrascolaire - littéraire ou universitaire, par exemple. On en voit un exemple assez frappant avec la biographie, genre très visible depuis longtemps en dehors de l'école, mais longtemps invisible dans les programmes et les manuels. Si la transposition de savoirs « savants » permet certes de légitimer et de rendre plus visibles certains genres, la visibilité scolaire de ces genres tient d'abord à l'usage scolaire qu' on peut en faire, comme je l'ai montré à propos des genres biographiques, mais aussi à propos de la tragédie ou des romans de Balzac. Autrement dit, ce sont les changements des finalités disciplinaires qui conduisent à changer les objets scolaires, et/ou à les reconfigurer.

\section{Pour conclure : discipline et culture scolaire}

Cette question du point de vue, si elle est intéressante pour approcher le genre, l'est d'ailleurs sans doute aussi pour approcher la question des genres littéraires : c'est en effet un angle, un point de vue, qui permet d'aborder à la fois la constitution des savoirs scolaires et leurs finalités, mais aussi la discipline et son histoire. Mon travail s'inscrit dans une réflexion sur les disciplines scolaires, ouverte par l'article fondateur de Chervel (1988/1998) qui avance l'idée d'une " existence autonome » (p. 17) des disciplines d'enseignement. J'ai ainsi cherché à questionner les genres littéraires et textuels non seulement en tant qu'ils peuvent être des objets d'enseignement et d'apprentissage (et donc susceptibles par exemple d'être déclinables en exercices), mais aussi en tant que leur scolarisation poursuit des finalités disciplinaires spécifiques, et produit des effets particuliers. L'ampleur de la périodisation choisie (de 1802 à nos jours) m'a conduite cependant à interroger, après bien d'autres chercheurs, l'idée d'une permanence de la discipline : non seulement les genres (corpus et notion) ont subi des variations importantes au cours de la période, mais les finalités disciplinaires elles-mêmes, telles qu'on peut les lire par exemple dans les textes institutionnels ou telles qu'elles transparaissent dans les discours des manuels, ont sensiblement évolué, voire se sont sensiblement modifiées. La discipline français a connu des variations trop importantes pour qu' on puisse les passer sous silence et les masquer sous une illusoire unité. J'ai donc utilisé, à la suite de LahanierReuter et Reuter (2007), le concept de « configuration disciplinaire », pour décrire les variations synchroniques et diachroniques ${ }^{(37)}$ de la discipline : il faut en effet prendre en compte différentes configurations disciplinaires ou prédisciplinaires pour comprendre la constitution et les enjeux de genres aussi divers que la tragédie classique, les textes fondateurs ou les romans de Balzac.

C'est ainsi qu'est apparu à plusieurs reprises ce qui m'apparaît comme une caractéristique essentielle de la discipline, et qui a déjà été souligné par de nombreux auteurs : le français est une discipline qui, même à des époques de rupture et de contestation (qu'il s'agisse de la fin du XIX ${ }^{\mathrm{e}}$ siècle ou des années 1970, par exemple), procède par «feuilletage » (Veck, 1990), par «sédimentation » (Schneuwly, 2007a), « par addition de couches successives » (Chervel, 1988/1998, p. 34). On pourrait employer la métaphore de la rénovation de bâtiment : le fonctionnement de la disci-

(37) J'ajoute donc la dimension diachronique, restée implicite dans Reuter (2007). 
pline fait que l'on sauvegarde l'ancien en accueillant le nouveau, c'est-à-dire qu'on bâtit du nouveau non pas seulement sur de l'ancien, mais avec de l'ancien, qu'il s'agisse de réemploi ou de recyclage - quitte d'ailleurs à détourner certains objets de leur usage initial. C'est ainsi que les genres rhétoriques (narration, description, etc.) sont recyclés en genres disciplinaires (narration et récits, descriptions et portraits, etc.), les extraits de tragédie classique utilisés comme modèle d'écriture, ou le roman autobiographique converti en autofiction pour les besoins d'un nouvel objet d'étude. C'est donc une culture scolaire en perpétuel mouvement, en quelque sorte, qui se dessine ainsi, culture faite de genres reconstruits et reconfigurés, de corpus textuels sélectionnés et scolarisés en vue d'objectifs spécifiques, et de vulgates scolaires sans cesse recomposées.

\section{Références bibliographiques}

ABASTADO, C. (1981): «La composition française et l'ordre du discours », Pratiques $\mathrm{n}^{\circ}$ 29, La Rédaction?, Metz, CRESEF, p. 3-18.

ANGLARD, V. (1992) : 50 modèles de commentaires composés, Alleur, Marabout.

AVIÉRINos, M., LABOURET, D. et PRAT, M.-H. (dir.) (2001) : Français $1^{\text {re }}$. Textes, mouvements, genres, méthodes, Paris, Bordas.

AVIÉRINOS, M. et PRAT, M.-H. (dir.) (1997) : Littérature. Textes, histoire, méthode (tome $2: \mathrm{XIX}^{\mathrm{e}}$ et $\mathrm{XX}^{\mathrm{e}}$ siècles), Paris, Bordas.

BAKHTINe, M. (1984) : Esthétique de la création verbale (traduction de A. Aucouturier), Paris, Gallimard (original publié en 1979).

BALIBAR, R. (1985) : L'institution du français, Paris, PUF.

BARTHES, R. (1970/2002) : «L'ancienne rhétorique », dans Barthes R. (2002), Euvres complètes, tome III, (1968-1971), Paris, Seuil, p. 527-601.

BATTEUX, C. (1780/1832): Chefs d'ouvre d'éloquence poétique à l'usage des jeunes orateurs, ou Discours français tirés des auteurs tragiques les plus célèbres, suivis d'une table raisonnée, dans laquelle on définit et on indique les différentes figures qui s'y rencontrent (nouvelle édition), Paris, Librairie classique de Maire-Nyon.

BAUtier, É. et GoigouX, R. (2004) : « Difficultés d'apprentissage, processus de secondarisation et pratiques enseignantes : une hypothèse relationnelle », Revue française de pédagogie $\mathrm{n}^{\circ} 148$, Évaluer et comprendre les effet des pratiques pédagogiques, Paris, INRP, p. 89-100.

BERniÉ, J.-P., JAUBERT, M. et REBIÈRE, M. (2008) : « Du contexte à la construction du sujet cognitif : 1'hypothèse énonciative », dans Brossard M. et Fijalkow J. (dir.), Vygotski et les recherches en éducation et en didactiques, Pessac, Presses Universitaires de Bordeaux, p. 123-141.

BoITEL, J. (1901) : Les meilleurs auteurs français du XVI au XIX siècle. Morceaux choisis de prose et de vers classés d'après les genres littéraires, Paris, Delagrave.

BraunschVig, M. (1921/1955) : Notre littérature étudiée dans les textes. II. Le $X V I I I^{e}$ et le XIX $X^{e}$ siècle ( $19^{\mathrm{e}}$ édition revue et augmentée), Paris, Armand Colin. 
CANVAT, K. (1998) : « La notion de genre à l'articulation de la lecture et de l'écriture », dans Reuter Y. (dir.), Les interactions lecture-écriture, Berne, Peter Lang, p. 243-262.

Chassang, A. et SENNINGER, Ch. (1966) : Recueil de textes littéraires français. XIX siècle, Paris, Hachette.

Chervel, A. (1988/1998) : «L'histoire des disciplines scolaires. Réflexions sur un domaine de recherche », Histoire de l'éducation n 38, Paris, INRP, p. 59-119, repris dans Chervel A. (1998), La culture scolaire. Une approche historique, Paris, Belin, p. 9-56.

— (2005) : «En quoi une culture peut-elle être scolaire? », dans Jacquet-Francillon F. et Kambouchner D. (dir.) (2005), La crise de la culture scolaire, Paris, PUF, p. 77-85.

— (2006) : Histoire de l'enseignement du français du XVII au XX siècle, Paris, Retz.

DAUNAY, B. (2003) : «Les liens entre écriture d'invention et écriture métatextuelle dans l'histoire de la discipline », Enjeux $\mathrm{n}^{\circ} 57$, Littérature et écriture d'invention, Namur, CEDOCEF, p. 9-24.

— (2004) : «Le commentaire : exercice, genre, activité ? », Les Cahiers Théodile $\mathrm{n}^{\circ}$ 5, Villeneuve d'Ascq, Université de Lille 3, p. 49-61.

Denizot, N. (2005) : «Classer la littérature au CDI », Recherches, ${ }^{\circ} 42$, Classer, Lille, ARDPF, p. 159-181.

— (2006a) : «Les "textes fondateurs" dans les programmes et manuels depuis 1938 : histoire et enjeux d'un corpus ", Le français aujourd'hui ${ }^{\circ} 155$, Lecture des textes fondateurs, Armand Colin-AFEF, p. 49-56.

— (2006b) : "Le biographique : vie et mort d'un genre scolaire », Recherches $\mathrm{n}^{\circ} 45$, Écritures de soi, Lille, ARDPF, p. 187-208.

- (2008) : Genres littéraires et genres textuels en classe de français : scolarisation, construction, fonctions et usages des genres dans la discipline français, Thèse de doctorat, Université de Lille 3.

- (2009a) : «Des "textes anciens" aux "textes fondateurs" en classe de sixième : enjeux socioculturels d'un corpus scolaire », dans B. Daunay, I. Delcambre et Y. Reuter (dir.), Didactique du français. Le socioculturel en question, Villeneuve d'Ascq, Presses du Septentrion, collection éducation et didactiques, p. 85-96.

- $(2009 \mathrm{~b})$ : «Un romancier classique d'une classe à l'autre : les extraits de Balzac dans les manuels scolaires ", Recherches $\mathrm{n}^{\circ} 50, D^{\prime}$ 'une classe à l'autre, Lille, ARDPF, p. 59-83.

- (2010) : "Construction d'un corpus scolaire : les "extraits" de Balzac dans les manuels scolaires (1880-2007) », dans B. Louichon et A. Rouxel (dir.), Du corpus scolaire à la bibliothèque intérieure, Presses Universitaires de Rennes, p. 81-90.

DOUAY-SOUBLIN, F. (1997) : «Les recueils de discours français pour la classe de rhétorique », dans Compère M.-M. et Chervel A. (dir.), Les humanités classiques, Paris, INRP, p. 151-185.

- (1999) : «La rhétorique en France au XIX ${ }^{\mathrm{e}}$ siècle à travers ses pratiques et ses institutions : restauration, renaissance, remise en cause » dans Fumaroli M. (dir.), Histoire de la rhétorique dans l'Europe moderne (1450-1950), Paris, PUF, p. 1071-1214.

DUfOUR, P. (1998) : Le réalisme, Paris, PUF.

FORESTIER, G. (2003) : Passions tragiques et règles classiques, Paris, PUF.

Genette, G. (1982) : Palimpsestes, Paris, Seuil. 
HUBERT, J. (2003) : Le romantisme. $2^{\text {de }}$, Paris, Magnard.

JACQUET-FRANCILLON, F. et KAMBOUCHNER, D. (dir.) (2005) : La crise de la culture scolaire, Paris, PUF.

JEY, M. (1998) : La littérature au lycée: Invention d'une discipline (1880-1925), Recherches textuelles $\mathrm{n}^{\circ}$ 3, Metz, Université de Metz.

KuENTZ, P. (1972) : «L'envers du texte», Littérature $\mathrm{n}^{\circ} 7$, Le discours de l'école sur les textes, Paris, Larousse, p. 3-26.

LAHANIER-REUTER, D. et REUTER, Y.(2007) : « L'analyse de la discipline : quelques problèmes pour la recherche en didactique ", dans Falardeau É., Fisher C., Simard C. et Sorin N. (dir.) La didactique du français. Les voies actuelles de la recherche, Laval, Presses de l'Université Laval, p. 27-42.

LANCREY-JAVAL, R. (dir.) (2004) : Littérature seconde, Paris, Hachette.

MARCOU, F.-L. (1884) : Morceaux choisis des classiques français (XVI ${ }^{e}, X V I I^{e}$, $X V I I I^{e}$ et XIX $X^{e}$ siècles) à l'usage des classes de sixième, cinquième et quatrième). Prosateurs ( $2^{\mathrm{e}}$ édition), Paris, Garnier Frères.

MitTerand, H. (dir.) (1986) : Littérature. Textes et documents. XIX siècle, Paris, Nathan.

NoËL, Fr. et DelaPlaCE, Fr. (1804/1805) : Leçons de littérature et de morale (vol. 1. Prose. vol. 2. Poésie ; $2^{\mathrm{e}}$ édition), Paris, Le Normant.

Petitjean, A. (2003) : "Histoire de l'écriture d'invention au lycée ", Pratiques $\mathrm{n}^{\circ}$ 117-118, Textes et valeurs, Metz, CRESEF, p. 181-207.

PRATIQUES (1987) : $\mathrm{n}^{\circ}$ 54, Les mauvais genres, Metz, CRESEF.

ReUter, Y. (1990) : «Définir les biens littéraires? », Pratiques n 67, Pratiques des textes littéraires, septembre 1990, Metz, CRESEF, p. 5-14.

— (2007) : «Disciplines scolaires », dans Reuter Y. (dir.), Dictionnaire des concepts fondamentaux de la didactique, Bruxelles, De Boeck, p. 85-89.

SCHNEUWLY, B. (2007a) : «Le "français": une discipline scolaire autonome, ouverte et articulée », dans Falardeau É., Fisher C., Simard C. et Sorin N. (dir.) La didactique du français. Les voies actuelles de la recherche, Laval, Presses de l'Université Laval, p. 9-26.

- (2007b) : «Genres écrits et oraux et forme scolaire. Enseignement et apprentissage de la langue première à l'école », dans Boré C. (dir.), Construire et exploiter des corpus de genres scolaires, Namur, Presses universitaires de Namur, p. 13-26.

VECK, B. (1990) : Trois savoirs pour une discipline, Paris, INRP. - (dir.) (1992) : Texte, thème, problématique. Morceaux choisis, composition française, listes d'oral, Paris, INRP.

VIALA, A. (1990) : «La dissertation fut d'abord un genre mondain snob », Pratiques $\mathrm{n}^{\circ}$ 68, La dissertation, Metz, CRESEF, p. 108-109.

VINCENT, G. (1980) : L'école primaire française, Lyon, Presses universitaires de Lyon/Éditions de la Maison des Sciences de l'homme. 